Pacific Journal of Mathematics

$C^{*}$-ALGEBRAS ASSOCIATED WITH FREE PRODUCTS O 


\title{
C*-ALGEBRAS ASSOCIATED WITH FREE PRODUCTS OF GROUPS
}

\section{William L. Paschke and Norberto Salinas}

\begin{abstract}
We show that the $C^{*}$-algebra of the left regular representation of the free product of two nontrivial groups, not both of order 2 , is simple and has a unique tracial state. In the case of the free product of cyclic groups, we investigate weak versus strong triviality for extensions of this $C^{*}$ algebra. One consequence of our extension-theoretic results is that the algebras of $n \times n$ matrices $(n=1,2, \cdots)$ over the $C^{*}$-algebra of the left regular representation of the free product of two cyclic groups are pairwise nonisomorphic.
\end{abstract}

For a discrete group $G$, we let $C_{r}^{*}(G)$ denote the $C^{*}$-algebra generated by the left regular representation of $G$ on $\ell^{2}(G)$. When $G$ is amenable, $C_{r}^{*}(G)$ is reasonably well-behaved. It coincides with the full group $C^{*}$-algebra $C^{*}(G)$ defined in 13.9 of [6], so that its *-representations on Hilbert space correspond precisely to the unitary representations of $G$, and is nuclear. (In fact, the conditions " $G$ amenable", " $C_{r}^{*}(G)=C^{*}(G)$ ", and " $C^{*}(G)$ (resp. $C_{r}^{*}(G)$ ) nuclear" are equivalent [7], [9], [4].) For certain nonamenable groups $G$, on the other hand, $C_{r}^{*}(G)$ possesses properties which in this context are somewhat pathological and which distinguish it quite sharply from $C^{*}(G)$. The latter algebra always admits a multiplicative linear functional, but R. T. Powers showed in [11] that $C_{r}^{*}(G)$ is simple and has a unique tracial state when $G$ is the free group $F_{2}$ on two generators. M. D. Choi [3] obtained the same properties for $C_{r}^{*}(G)$ when $G$ is the free product $\boldsymbol{Z}_{2} * \boldsymbol{Z}_{3}$ of the cyclic groups of orders 2 and 3. Choi also showed that although $C_{r}^{*}\left(\boldsymbol{Z}_{2} * \boldsymbol{Z}_{3}\right)$ is nonnuclear, it (and therefore $C_{r}^{*}\left(F_{2}\right)$ ) can be embedded in a nuclear $C^{*}$-algebra. By a recent result of $\mathrm{S}$. Wassermann [14], however, $C^{*}\left(F_{2}\right)$ is not a $C^{*}$-subalgebra of any nuclear $C^{*}$-algebra.

Our main result in $\S 1$ below is that $C_{r}^{*}\left(G_{1} * G_{2}\right)$ is simple and has a unique tracial state whenever $G_{1}$ and $G_{2}$ are nontrivial groups not both of order 2. This subsumes the cases treated in [3] and [11]. Generalizing somewhat the example in [3], we also show that if $U$ and $V$ are unitary operators of orders 2 and $n(\geqq 3)$, respectively, for which there exists a projection $P$ satisfying $P+U P U^{*}=I=$ $P+V P V^{*}+V^{2} P\left(V^{*}\right)^{2}+\cdots+V^{n-1} P\left(V^{*}\right)^{n-1}$, then $C^{*}(U, V)$ is naturally isomorphic to $C_{r}^{*}\left(\boldsymbol{Z}_{2} * \boldsymbol{Z}_{n}\right)$. In $\S 2$, we introduce a numerical invariant $j(A)$ for separable unital $C^{*}$-algebras $A$; namely, $j(A)$ is the nonnegative integer that generates the subgroup of $Z$ consisting of 
the indices of all unitaries in the Calkin algebra that commute with the image of $A$ under a trivial extension. We prove that $j\left(C_{r}^{*}\left(\boldsymbol{Z}_{n} * \boldsymbol{Z}_{n}\right)\right)$ divides $n$ for $n=1,2, \cdots$, and deduce from this that $j\left(C_{r}^{*}(F)\right)=1$ if $F$ is free on a finite or countably infinite set of generators. In other words, all weakly trivial extensions of $C_{r}^{*}(F)$ are strongly trivial. Another consequence of our result for $C_{r}^{*}\left(\boldsymbol{Z}_{n} * \boldsymbol{Z}_{n}\right)$ is that the $C^{*}$ algebras $C_{r}^{*}(G) \otimes M_{n}(n=1,2, \cdots)$ are pairwise nonisomorphic whenever $G$ is the free product of two cyclic groups.

1. Simplicity and uniqueness of trace. Given a (discrete) group $G$, we shall denote by $\ell^{2}(G)$ the Hilbert space of all absolutely squaresummable functions $f: G \rightarrow C$. The left regular representation of $G$ on $\ell^{2}(G)$ is obtained by defining, for each $s$ in $G$, the unitary operator $L_{s}$ by $\left(L_{s} f\right)(t)=f\left(s^{-1} t\right)$ ( $t$ in $\left.G\right)$. Notice that $L_{s} \delta_{t}=\delta_{s t}$, where $\delta_{t}$ is the characteristic function of $\{t\}$. The linear span of $\left\{L_{s}: s \in G\right\}$ is a *algebra, which we denote by $A^{*}(G)$. We write $C_{r}^{*}(G)$ for the norm-closure of $A^{*}(G)$. There is a natural faithful tracial state $\tau$ on $C_{r}^{*}(G)$ defined by $\tau(T)=\left(T \delta_{e}, \delta_{e}\right)$, where $e$ is the identity of $G$. We shall be interested in $C_{r}^{*}(G)$ when $G$ is a free product. By definition [8], the free product $G_{1} * G_{2}$ of two groups $G_{1}$ and $G_{2}$ is the set consisting of the empty word (denoted by $e$ ) together with all reduced words $w=a_{1} a_{2} \cdots a_{n}$, where the $a_{j}$ 's are elements of either $G_{1}$ or $G_{2}$ different from the identity and satisfy the condition: $a_{j} \in G_{i}$ implies $a_{j+1} \in G_{3-i}(1 \leqq j \leqq n-1, i=1,2)$. The integer $n=\ell(w)$ is called the length of the word $w$. We set $\ell(e)=$ 0 . The set $G_{1} * G_{2}$ becomes a group with identity $e$ if we multiply reduced words by juxtaposing them and then performing the obvious simplifications required to put the product in reduced form.

The proof of the following theorem is based on the techniques of [11], and generalizes the results of [11] and [3].

THEOREM 1.1. Let $G=G_{1} * G_{2}$, where $G_{1}$ and $G_{2}$ are nontrivial groups not both of order 2 . Then

(a) $C_{r}^{*}(G)$ is nonnuclear;

(b) $C_{r}^{*}(G)$ is simple;

(c) $\tau$ is the only tracial state on $C_{r}^{*}(G)$.

Proof. For definiteness, assume that $G_{2}$ has order greater than 2. Let $a$ in $G_{1}$ and $b, c$ in $G_{2}$ be such that none of the elements $a$, $b, c, b^{-1} c$ is the identity. Part (a) follows from the simple observation that $G$ contains a copy of $F_{2}$. Indeed, let $r=a b$ and $s=a c$. It readily follows that $r^{2}$ and $s^{2}$ generate a copy of $F_{2}$. Therefore, we deduce that $G$ is nonamenable, and from [9] we conclude that $C_{r}^{*}(G)$ is nonnuclear. Now, as in [11], parts (b) and (c) are direct conse- 
quences of the following lemma.

LeMma 1.2. Let $G=G_{1} * G_{2}$ as above, and let $S$ be a self-adjoint operator in $C_{r}^{*}(G)$. Then $\tau(S) I$ belongs to the norm closure of the convex hull of the unitary orbit of $S$.

Proof. As in [11], it suffices to prove the lemma when $S$ belongs to $A^{*}(G)$ and $\tau(S)=0$. In this case, $S$ can be written as

$$
S=\sum_{i=1}^{n}\left(\alpha_{i} L_{x_{i}}+\bar{\alpha}_{i} L_{x_{i}^{-1}}\right),
$$

where the $\alpha_{i}$ 's are scalars and each $x_{i}$ is different from $e$. We set $r=a b$ as above and $j=1+\max \left\{\ell\left(x_{1}\right), \cdots, \ell\left(x_{n}\right)\right\}$. Consider $r^{j} x_{i}^{-1} r^{-j}$ for $1 \leqq i \leqq n$. If $x_{i}$ is a power of $r$, then of course $r^{j} x_{i}^{-1} r^{-j}=x_{i}^{-1}$. Otherwise, it is easy to see that $r^{j} x_{i}^{-1} r^{-j}$ begins with a positive power of $r$ and ends with a negative power of $r$, that is, $r^{j} x_{i}^{-1} r^{-j}$ can be written as $r t$ and as $t^{\prime} r^{-1}$, where $t$ begins with an element of $G_{1} \backslash\{e\}$ and $t^{\prime}$ ends with an element of $G_{1} \mid\{e\}$. For $k=1,2, \cdots$, let $z_{k}=r^{k} s^{2} r^{j}$, where $s=a c$ is as before. Further, let $A_{k}$ be the set of all reduced words $w$ such that (the reduced form of) $r^{-k} w$ begins with $a$, but $a^{-1} r^{-k} w$ does not begin with $b$. One readily checks that the $A_{k}$ 's are pairwise disjoint. We claim also that if $y \in G \backslash A_{k}$, then $s^{-1} r^{-k} y$ begins with an element of $G_{2} \backslash\{e\}$. (If $r^{-k} y$ does not begin with $a$, then $s^{-1} r^{-k} y=c^{-1} a^{-1} r^{-k} y$ begins with $c^{-1}$. If $a^{-1} r^{-k} y$ begins with $b$, then $s^{-1} r^{-k} y$ begins with $c^{-1} b$.) Hence, $s^{-2} r^{-k} y$ begins with $c^{-1}$ for all $y$ in $G \backslash A_{k}$. We let $P_{k}$ be the projection of $\ell^{2}(G)$ onto the subspace $\mathfrak{M}_{k}$ consisting of all those functions $f$ whose support $\operatorname{supp}(f)$ is contained in $A_{k}$. Because the $A_{k}$ 's are disjoint, the $P_{k}$ 's are orthogonal to one another. We claim that the compression of $L_{z_{k}} S L_{z_{k}^{-1}}$ to $\mathfrak{M}_{k}$ is zero for $k \geqq 1$. To see this, it suffices to show that $\left(I-P_{k}\right) L_{z_{k} x_{i} z_{k}^{-1}}\left(I-P_{k}\right)=0$ for $1 \leqq i \leqq n$. Thus, given a function $f$ in $\ell^{2}(G)$ supported in $G \backslash A_{k}$, we must show that $f\left(z_{k} x_{i}^{-1} z_{k}^{-1} y\right)=0$ for every $y$ in $G \backslash A_{k}$. This is the case if for $y$ in $G \backslash A_{k}$, we have $z_{k} x_{i}^{-1} z_{k}^{-1} y$ in $A_{k}$. But this follows easily from our observation above that $s^{-2} r^{-k} y$ begins with $c^{-1}$, for if $x_{i}^{-1}$ is a positive power of $r$, the only cancellation that occurs in $z_{k} x_{i}^{-1} z_{k}^{-1} y=r^{k} s^{2} x_{i}^{-1} s^{-2} r^{-k} y$ is at and possibly to the left of the final $b$ in $x_{i}^{-1}$. Otherwise, $r^{j} x_{i}^{-1} r^{-j}$ is either a negative power of $r$ or begins with a positive power of $r$ and ends with a negative power of $r$; in these cases, no cancellation can occur in $z_{k} x_{i}^{-1} z_{k}^{-1} y=r^{k} s\left(s r^{j} x_{i}^{-1} r^{-j}\right)\left(s^{-2} r^{-k} y\right)$ except strictly inside the expressions $s r^{j} x_{i}^{-1} r^{-j}$ and $s^{-2} r^{-k} y$. This establishes our claim about $L_{z_{K}} S L_{z_{k}^{-1}}$. Our next observation is that if $P$ is any projection and $T$ any bounded operator on a Hilbert space $\mathfrak{S}$ such that $(I-P) T(I-P)=0$, then $|(T f, f)| \leqq 2\|T\|\|P f\|$ for all $f$ in $\mathscr{S}$ with 
$\|f\| \leqq 1 . \quad$ Indeed,

$$
\begin{aligned}
|(T f, f)| & \leqq|(T f, P f)|+|(T f,(I-P) f)| \\
& =|(T f, P f)|+|(T P f,(I-P) f)| \\
& \leqq 2\|T\|\|P f\| .
\end{aligned}
$$

We apply this remark to our operator $S$ and the projections $P_{k}$ to deduce that for every $f$ in the unit ball of $\ell^{2}(G)$, we have

$$
\begin{aligned}
\left|\left(\left(\frac{1}{9} \sum_{k=1}^{9} L_{z_{k}} S L_{z_{k}^{-1}}\right) f, f\right)\right| & \leqq \frac{1}{9} \sum_{k=1}^{9}\left|\left(L_{z_{k}} S L_{z_{k}-1} f, f\right)\right| \\
& \leqq \frac{1}{9} \sum_{k=1}^{9} 2\|S\|\left\|P_{k} f\right\| \\
& \leqq \frac{2}{9}\|S\| \sqrt{9}\left(\sum_{k=1}^{9}\left\|P_{k k} f\right\|^{2}\right)^{1 / 2} \\
& \leqq \frac{2}{3}\|S\| .
\end{aligned}
$$

Since $\sum_{k=1}^{9} L_{z_{k}} S L_{z_{k}^{-1}}$ is self adjoint, we obtain the following inequality:

$$
\left\|\frac{1}{9} \sum_{k=1}^{9} L_{z_{k}} S L_{z_{k}^{-1}}\right\| \leqq \frac{2}{3}\|S\| \text {. }
$$

Now by iterating (*) as many times as necessary, we can produce a self adjoint operator of trace zero in the convex hull of the unitary orbit of $S$ whose norm is arbitrarily small.

Remark 1.3. (a) We are indebted to Vern Paulsen for a helpful comment that enabled us to reduce our original constant 79/81 to $2 / 3$ in the above estimate $\left(^{*}\right)$. Indeed, we could have taken only five terms in the above argument and ended up with a constant $2 / \sqrt{5}$ which is still less than 1 and suitable for our purpose.

(b) It is easy to see that the converse of Theorem 1.1 also holds. That is, if $G=Z_{2} * Z_{2}$, then $G$ is not i.c.c. and hence $C_{r}^{*}(G)$ has nontrivial center. This means that $C_{r}^{*}(G)$ can neither be simple nor have a unique tracial state. Furthermore, it can be easily seen that $\boldsymbol{Z}_{2} * \boldsymbol{Z}_{2}$ is an amenable group and hence $C_{r}^{*}\left(\boldsymbol{Z}_{2} * \boldsymbol{Z}_{2}\right)$ is a nuclear $C^{*}$-algebra.

(c) The fact that $C_{r}^{*}\left(G_{1} * G_{2}\right)$ is simple (for $G_{1}$ and $G_{2}$ as in Theorem 1.1) implies that $C_{r}^{*}\left(G_{1} * G_{2}\right)$ cannot coincide with the $C^{*}$ algebraic free product of $C_{r}^{*}\left(G_{1}\right)$ and $C_{r}^{*}\left(G_{2}\right)$ (cf. [1]). On the other hand, full group $C^{*}$-algebras satisfy $C^{*}\left(G_{1} * G_{2}\right)=C^{*}\left(G_{1}\right) * C^{*}\left(G_{2}\right)$ because of the naturality of both free products.

(d) Since $F_{2}$ coincides with $Z * Z$, Theorem 1.1 generalizes the result of [11]. Moreover, the group $G$ considered in [3] can also be 
defined as $G=\boldsymbol{Z}_{2} * \boldsymbol{Z}_{3}$, so Theorem 1.1 also extends the result of [3].

Notice that one can generalize the $C^{*}$-algebra $C^{*}(u, v)$ considered in [3] as follows. Let $\pi_{1}$ and $\pi_{2}$ be ${ }^{*}$-representations of the matrix algebras $M_{2}$ and $M_{n}(n \geqq 3)$ on an infinite-dimensional Hilbert space for which the projection

$$
P=\pi_{1}\left(\begin{array}{ll}
1 & 0 \\
0 & 0
\end{array}\right)
$$

coincides with the projection

$$
\pi_{2}\left(\begin{array}{cccc}
1 & 0 & \cdots & 0 \\
0 & 0 & \cdots & 0 \\
\vdots & \vdots & & \\
0 & 0 & \cdots & 0
\end{array}\right)
$$

Let

$$
U=\pi_{1}\left(\begin{array}{ll}
0 & 1 \\
1 & 0
\end{array}\right) \text { and } V=\pi_{2}\left(\begin{array}{ccccc}
0 & \cdots & \cdots & 0 & 1 \\
1 & 0 & \cdots & \cdots & 0 \\
0 & 1 & 0 & \cdots & 0 \\
\vdots & & & & 0 \\
0 & \cdots & 0 & 1 & 0
\end{array}\right)
$$

Then

$$
\begin{aligned}
& U^{*}=U^{-1}, \quad V^{*}=V^{-1}, \quad U^{2}=I=V^{n}, \quad \text { and } \\
& P+U P U^{*}=I=\sum_{i=0}^{n-1} V^{i} P\left(V^{*}\right)^{i} .
\end{aligned}
$$

From [10], it follows that $C^{*}(P, U, V)$ is isomorphic to $\mathscr{O}_{n-1} \otimes M_{2}$, where $\mathscr{O}_{k}$ (for $k \geqq 2$ ) is the $C^{*}$-algebra generated by $k$ isometries studied in [5]. Since this identification is a natural one, it also follows that $C^{*}(U, V)$ is algebraically unique in the sense that if $P^{\prime}$ is any other projection and $U^{\prime}$ and $V^{\prime}$ are any other two operators satisfying (**), then there is a *-isomorphism of $C^{*}\left(U^{\prime}, V^{\prime}\right)$ with $C^{*}(U, V)$ sending $U^{\prime}$ to $U$ and $V^{\prime}$ to $V$. In particular, $U$ and $V$ can be represented spatially as described in [10].

Another consequence of the $C^{*}$-algebra uniqueness of $C^{*}(U, V)$ is the following theorem.

THEOREM 1.4. Let $U$ and $V$ be as above. Then $C^{*}(U, V)$ is *-isomorphic to $C_{r}^{*}\left(\boldsymbol{Z}_{2} * \boldsymbol{Z}_{n}\right)$, and hence is nonnuclear and simple, and has a unique tracial state.

Proof. Let $G=\boldsymbol{Z}_{2} * \boldsymbol{Z}_{n}$, and let $a$ in $\boldsymbol{Z}_{2}, b$ in $\boldsymbol{Z}_{n}$ be the basic 
generators. Let $A$ be the subset of $G$ consisting of all words that begin with $a$. Then $G$ is the disjoint union of $A$ and $a A$, and the sets $A, b A, \cdots, b^{n-1} A$ are pairwise disjoint. Furthermore,

$$
G \backslash \bigcup_{i=0}^{n-1} b^{i} A=\left\{e, b, \cdots, b^{n-1}\right\} .
$$

Now let $\mathfrak{\mathcal { C }}=\ell^{2}(G), U^{\prime}=L_{a}, V^{\prime}=L_{b}$, and let $P^{\prime}$ be the projection on the subspace of $\mathfrak{F}$ consisting of all those functions $f$ such that $\operatorname{supp}(f) \subseteq A$. Then all of the requirements of $\left(^{* *}\right)$ are satisfied except the last. Indeed, $I-\sum_{i=1}^{n-1}\left(V^{\prime}\right)^{i} P\left(V^{\prime *}\right)^{i}$ is the $n$-dimensional projection onto the subspace of functions supported in $\left\{e, b, \cdots, b^{n-1}\right\}$. Therefore, all the properties of $\left({ }^{*}\right)$ actually hold in the Calkin algebra. The restriction of the Calkin map to $C^{*}\left(U^{\prime}, V^{\prime}\right)$ is an isomorphism, so $C^{*}\left(U^{\prime}, V^{\prime}\right)=C_{r}^{*}\left(\boldsymbol{Z}_{2} * \boldsymbol{Z}_{n}\right)$ is canonically isomorphic to $C^{*}(U, V)$ (via the Calkin algebra).

REMARK 1.5. (a) With a small extra effort, one can actually construct a subset $A$ of $G\left(=\boldsymbol{Z}_{2} * \boldsymbol{Z}_{n}\right)$ in the above proof so that the projection $P^{\prime}$ defined from $A$ together with the unitaries $U^{\prime}$ and $V^{\prime}$ satisfy all of the requirements of $\left(^{* *}\right)$. Indeed, one can define such a set $A$ by induction on the length of reduced words in $G$ as follows. Start with $e \notin A$; inductively, if $w=x z$ is a reduced word with $\zeta(w)=n+1$ and $\ell(z)=n$, then $w \in A$ if and only if $z \notin A$ and $x$ is either $a$ or $b$.

(b) One can actually define intrinsically the trace $\tau$ on $C^{*}(U, V)$. In fact, we first notice that every operator in $A^{*}(G)$ can be written as $T=\alpha_{0} I+\sum_{i=1}^{n} \alpha_{i} L_{x_{i}}$ where $x_{i} \neq e$ for $1 \leqq i \leqq n$ and $\alpha_{0}$ is uniquely determined by $\alpha_{0}=\tau(T)$. Let $\phi$ be the isomorphism from the group $\left\{L_{x}: x \in G\right\}$ onto the group generated by $U$ and $V$ determined by $\phi\left(L_{a}\right)=U$ and $\phi\left(L_{b}\right)=V$. Then the correspondence

$$
T \rightarrow \alpha_{0} I+\sum_{i=1}^{n} \alpha_{i} \phi\left(L_{x_{i}}\right)
$$

establishes a *-isomorphism from $A^{*}(G)$ onto the *-algebra generated by $U$ and $V$. This isomorphism extends to the canonical isomorphism from $C_{r}^{*}(G)$ onto $C^{*}(U, V)$. Thus, one can define, on the *algebra generated by $U$ and $V$, a trace $\tau$ by

$$
\tau\left(\alpha_{0} I+\sum_{i=1}^{n} \alpha_{i} \phi\left(L_{x_{i}}\right)\right)=\alpha_{0},
$$

which determines the trace on $C^{*}(U, V)$.

(c) As in [3], it can be proved that every operator in $C^{*}(U, V)$ is quasi-triangular, but $U$ and $V$ are not simultaneously quasi-triangular. 
When $G$ is as in Theorem 1.1, one can show without much difficulty that the conjugacy class of every element in $G \backslash\{e\}$ contains two elements which generate a copy of $F_{2}$ in $G$. Thus, $G$ has no nontrivial amenable normal subgroups. The following proposition shows that conclusions (b) and (c) of Theorem 1.1 fail for groups $G$ which do have a nontrivial amenable normal subgroup.

Proposition 1.6. Let $G$ be a group with amenable normal subgroup $H$. There is a tracial state $\tau_{1}$ on $C_{r}^{*}(G)$ such that $\tau_{1}\left(L_{x}\right)=1$ for every $x$ in $H$.

Proof. First, let $H$ be any subgroup of $G$. The decomposition of $G$ into right $H$-cosets decomposes the representation $x$ (in $H) \rightarrow$ $L_{x}$ (in $\mathscr{L}\left(\ell^{2}(G)\right)$, the algebra of bounded operators on $\ell^{2}(G)$ ) into the direct sum of copies of the left regular representation, whence it follows that the $C^{*}$-subalgebra of $C_{r}^{*}(G)$ generated by $\left\{L_{x}: x \in H\right\}$ is *-isomorphic to $C_{r}^{*}(H)$. We shall thus regard $C_{r}^{*}(H)$ as a *-subalgebra of $C_{r}^{*}(G)$. Let $S$ be a finite linear combination of $L_{x}$ 's $(x$ in $H)$ and $T$ a finite linear combination of $L_{y}$ 's $(y$ in $G \backslash H)$. If $f$ and $g$ are unit vectors in $\ell^{2}(G)$ supported in $H$, then $(T f, g)=0$ and $\|S+T\| \geqq$ $|((S+T) f, g)|=|(S f, g)|$, so we have $\|S+T\| \geqq\|S\|$. It follows that there is a conditional expectation $E: C_{r}^{*}(G) \rightarrow C_{r}^{*}(H)$ such that $E\left(L_{x}\right)=L_{x}$ (resp. 0) when $x \in H$ (resp. $x \in G \backslash H$ ). Now suppose that $H$ is an amenable normal subgroup of $G$. By 3.5.2 of [7], every unitary representation of $H$ extends to a *-representation of $C_{r}^{*}(H)$. In particular, the trivial representation of $H$ on $C$ extends to a multiplicative linear functional $\phi$ on $C_{r}^{*}(H)$ such that $\phi\left(L_{x}\right)=1$ for every $x$ in $H$. Let $\tau_{1}=\phi \circ E$, so $\tau_{1}$ is a state of $C_{r}^{*}(G)$ with $\tau_{1}\left(L_{x}\right)=1$ for every $x$ in $H$. For $s, t$ in $G$, we have $s t \in H$ if and only if $t s \in H$ (since $H$ is a normal subgroup) and hence $\tau_{1}\left(L_{s} L_{t}\right)=\tau_{1}\left(L_{t} L_{s}\right)=1$ (resp. 0) if $s t \in H$ (resp. st $\notin H$ ). It now follows easily that $\tau_{1}$ is tracial.

We have shown that the natural trace is not the only tracial state on $C_{r}^{*}(G)$ when $G$ contains an amenable normal subgroup $H$ different from $\{e\}$. Notice also that $\left\{T \in C_{r}^{*}(G): \tau_{1}\left(T^{*} T\right)=0\right\}$ is a proper ideal of $C_{r}^{*}(G)$ containing $1-L_{x}$ for every $x$ in $H$, so $C_{r}^{*}(G)$ is not simple under these circumstances either.

2. Weakly trivial extensions. By an extension of a separable unital $C^{*}$-algebra $A$, we mean a unital *-monomorphism from $A$ into the Calkin algebra $\mathscr{Q}(\mathfrak{S})$ of a separable infinite-dimensional Hilbert space $\mathfrak{F}$. Two extensions $\sigma, \tau: A \rightarrow \mathscr{Q}(\mathfrak{S}), \mathbb{Q}\left(\mathfrak{S}^{\prime}\right)$ are said to be strongly equivalent if there is a unitary $U: \mathfrak{S} \rightarrow \mathscr{S C}^{\prime}$ such that $\tau$ is the composition of $\sigma$ with the *-isomorphism from $\mathscr{Q}(\mathfrak{S})$ to $\mathscr{Q}\left(\mathfrak{S E}^{\prime}\right)$ induced 
by $U$. (The corresponding notion of weak equivalence requires only that $U$ be a partial isometry with finite-dimensional kernel and cokernel.) We write [ $\tau]$ for the strong equivalence class of the extension $\tau$. Let $A$ be faithfully and unitally represented on $\mathfrak{K}$ with $A$ containing no nonzero compact operators, and for $r=0,1,2, \cdots$, let $\tau_{r}: A \rightarrow \mathbb{Q}\left(\tilde{S}_{r}\right)$ be the extension of $A$ obtained by compression to a closed subspace $\mathfrak{K}_{r}$ of codimension $r$ in $\mathscr{S}_{\mathfrak{C}}$ followed by the Calkin map $\mathscr{L}\left(\mathscr{S}_{r}\right) \rightarrow \mathscr{Q}\left(\mathscr{S}_{r}\right)$. For each $r$, the strong equivalence class $\left[\tau_{r}\right]$ is independent of the representation of $A$ on $\mathfrak{S}$ by 1.5 of [13] and independent of the choice of $\mathfrak{F}_{r}$ by elementary arguments. In particular, $\tau_{0}$ is, up to strong equivalence, the only extension of $A$ that factors through a *-representation of $A$ on the underlying Hilbert space. We call such extensions strongly trivial. Each of the $\tau_{r}$ 's is weakly equivalent to $\tau_{0}$, that is, weakly trivial. Together with their inverses relative to the usual semigroup operation "+" on strong equivalence classes, the $\left[\tau_{r}\right]$ 's comprise all of the weakly trivial classes.

We let $j(A)$ be the smallest positive integer $j$ such that $\tau_{j}$ is strongly trivial, setting $j(A)=0$ if $\tau_{j}$ is not strongly trivial for any $j \geqq 1$. It is clear from our comments above that $j(A)$ is an algebraic invariant of $A$.

REMARKs 2.1. (a) We have $\tau_{k}$ strongly trivial if and only if $j(A)$ divides $k$. (This is because $\left[\tau_{r}\right]+\left[\tau_{s}\right]=\left[\tau_{r+s}\right]$ for every $r, s \geqq 0$.)

(b) If $B$ is a unital $C^{*}$-subalgebra of $A$, then $j(B)$ divides $j(A)$. (This follows from the observation that $\tau_{r}$ (for $B$ ) is just the restriction of $\tau_{r}$ (for $A$ ) to $B$.)

(c) If $u$ is a unitary in $\mathbb{Q}(\mathfrak{S})$ of index $r(\geqq 0)$, then $\tau_{r}$ is strongly equivalent to the extension $u \tau_{0}(\cdot) u^{*}$. In order for this extension to be strongly equivalent to $\tau_{0}$, there must be a unitary $v$ of index 0 in $\mathscr{Q}(\mathfrak{S})$ such that $v u \tau_{0}(\cdot) u^{*} v^{*}=\tau_{0}(\cdot)$. Hence, $j(A)$ is the nonnegative generator of the subgroup of $\boldsymbol{Z}$ consisting of the indices of all unitaries in the Calkin algebra which commute with the image of $A$ under a trivial extension.

By way of examples, we mention that $j(A)=1$ whenever $A$ is commutative (1.5 of [2]) and that $j\left(M_{n}\right)=n$ by Remark 2.1 (c) above. More generally, it follows from 3.3 of [12] that if $A$ has an $n$ dimensional irreducible representation, then $j(A)$ divides $n$. Thus for full group $C^{*}$-algebras $C^{*}(G)$ (when $G$ is countable), we have $j\left(C^{*}(G)\right)=1$. On the other hand, $j\left(\mathcal{O}_{n}\right)=0(n \geqq 2)[10]$, where $\mathcal{O}_{n}$ is the algebra studied in [5]. One of our results in this section is that $j\left(C_{r}^{*}(F)\right)=1$ whenever $F$ is the free group on a finite or countably infinite set of generators. We will show this by showing that 
$j\left(C_{r}^{*}\left(\boldsymbol{Z}_{n} * \boldsymbol{Z}_{n}\right)\right)$ divides $n$ for $n \geqq 2$ and then observing that $C_{r}^{*}(\boldsymbol{F})$ embeds unitally in (for instance) $C_{r}^{*}\left(\boldsymbol{Z}_{3} * \boldsymbol{Z}_{3}\right)$ and $C_{r}^{*}\left(\boldsymbol{Z}_{4} * \boldsymbol{Z}_{4}\right)$.

THEOREM 2.2. Let $G=Z_{n} * Z_{n}$, where $n \geqq 2$. The extension of $C_{r}^{*}(G)$ obtained by compression to a subspace of $\ell^{2}(G)$ of codimension $n$ followed by the Calkin map is strongly trivial. Thus, $j\left(C_{r}^{*}(G)\right.$ ) divides $n$.

Proof. Let $b$ and $c$ in $G$ be the generators of the two copies of $Z_{n}$ of which $G$ is the free product. For a reduced word $w$ in $G \backslash\{e\}$, let $D(w)$ be the set of all reduced words of the form $y w$ where $y$ is either $e$ or a word ending in a nonzero power of $b$ (resp. c) if $w$ begins with a nonzero power of $c$ (resp. $b$ ). Said another way, $D(w)$ is the set of all words that "end in $w$ ". Let

$$
E=\bigcup\left\{D\left(b^{k} c^{j}\right): 1 \leqq k \leqq n-1,0 \leqq j \leqq n-1\right\},
$$

and let $\AA$ be the subspace of $\ell^{2}(G)$ consisting of the functions supported in $E$. Since $G \backslash E=\left\{e, c, c^{2}, \cdots, c^{n-1}\right\}$, the subspace $\Omega$ has codimension $n$. We will exhibit a ${ }^{*}$-representation $\theta$ of $C_{r}^{*}(G)$ on $\Re$ such that $\theta\left(L_{b}\right)$ and $\theta\left(L_{c}\right)$ differ finite-dimensionally from the compressions of $L_{b}$ and $L_{c}$ to $\Re$, thereby establishing the strong triviality of the extension of $C_{r}^{*}(G)$ obtained from compression to $\Omega$. For $1 \leqq$ $j \leqq n-1$, consider

$$
E_{j}=D\left(b^{j}\right) \cup \bigcup\left\{D\left(b^{k} c^{j}\right): 1 \leqq k \leqq n-1\right\}
$$

and let $\Re_{j}$ be the subspace of $\ell^{2}(G)$ consisting of functions supported in $E_{j}$. Since $E$ is the disjoint union of the $E_{j}{ }^{\prime}$ 's, $\Omega$ is the direct sum of the $\mathscr{R}_{j}$ 's. Further, let

$$
\begin{aligned}
& B=\bigcup\left\{D\left(b^{k}\right): 1 \leqq k \leqq n-1\right\} \text { and } \\
& C=\{e\} \cup \bigcup\left\{D\left(c^{j}\right): 1 \leqq j \leqq n-1\right\},
\end{aligned}
$$

so that $G$ is the disjoint union of $B$ and $C$. For $1 \leqq j \leqq n-1$, multiplication on the right by $c^{j}$ maps $B$ onto

$$
\bigcup\left\{D\left(b^{k} c^{j}\right): 1 \leqq k \leqq n-1\right\},
$$

while right multiplication by $b^{j}$ maps $C$ onto $D\left(b^{j}\right)$. We may thus define unitaries $V_{j}: \ell^{2}(G) \rightarrow \AA_{j}$ on basis vectors by setting $V_{j} \delta_{w}=\delta_{w c}$ (resp. $\delta_{w b j}$ ) if $w \in B$ (resp. $w \in C$ ). These give rise to *-representations $\theta_{j}: C_{r}^{*}(G) \rightarrow \mathscr{L}\left(\Re_{j}\right)$ defined by $\theta_{j}(T)=V_{j} T V_{j}^{*}$. Let $\theta$ be the direct sum of the $\theta_{j}$ 's, so $\theta$ is a *-representation of $C_{r}^{*}(G)$ on $\Omega$. We claim that $\theta_{j}\left(L_{b}\right) V_{j} \delta_{w}=L_{b} V_{j} \delta_{w}$ for every $w$ in $G \backslash\left\{e, b, b^{2}, \cdots, b^{n-1}\right\}$. If such a $w$ belongs to $B$ (that is, $w$ ends with a nonzero power of $b$ preceded by a nonzero power of $c$ ), then so does $b w$ and hence 
$\theta_{j}\left(L_{b}\right) V_{j} \delta_{w}=V_{j} \delta_{b w}=\delta_{b w c}=L_{b} V_{j} \delta_{w}$. If, on the other hand, $w$ belongs to $C$ (that is, $w$ ends with a nonzero power of $c$ ), then $b w$ belongs to $C$ also and we have $V_{j} \delta_{b w}=\delta_{b w b^{j}}=L_{b} V_{j} \delta_{w}$. It follows that $\theta\left(L_{b}\right)$ differs from the compression of $L_{b}$ to $\Omega$ by a finite-dimensional operator of rank (at most) $n(n-1)$. In similar fashion, one checks that $\theta\left(L_{c}\right) V_{j} \delta_{w}=L_{c} V_{j} \delta_{w}$ for every $w$ in $G$, using the observation that $c w \in B$ if and only if $w \in B$. This shows that $\theta\left(L_{c}\right)$ coincides with the compression of $L_{c}$ to $\Omega$, completing the proof of the lemma.

We now explore some consequences of this theorem.

THEOREM 2.3. Let $F$ be the free group on a finite or countably infinite set of generators. Then $j\left(C_{r}^{*}(F)\right)=1$, that is, all weakly trivial extensions of $C_{r}^{*}(F)$ are strongly trivial.

Proof. Let $F$ be freely generated by the (finite or countably infinite) set $\left\{a_{1}, a_{2}, \cdots\right\}$. Take $n \geqq 3$ and let $b$ and $c$ be the generators of the copies of $\boldsymbol{Z}_{n}$ of which $\boldsymbol{Z}_{n} * \boldsymbol{Z}_{n}$ in the free product. We may embed $F$ in $\boldsymbol{Z}_{n} * \boldsymbol{Z}_{n}$ by (for example) sending each $a_{k}$ to $(c b)^{k} c(c b)^{k}$, so as in the proof of Proposition 1.6 above, $C_{r}^{*}(F)$ embeds unitally in $C_{r}^{*}\left(\boldsymbol{Z}_{n} * \boldsymbol{Z}_{n}\right)$. The theorem now follows from Theorem 2.2 and Remark 2.1 (b).

Since $j\left(M_{n}\right)=n$, the theorem above and Remark 2.1 (b) imply that $C_{r}^{*}(F)$ cannot unitally contain a copy of $M_{n}$ for any $n \geqq 2$. (It has long been conjectured, at least for $F=F_{2}$, that in fact $C_{r}^{*}(F)$ contains no projections except 0 and $I$.) One consequence of the following proposition is that the $C^{*}$-algebras $C_{r}^{*}(F) \otimes M_{n}(n=1,2, \cdots)$ are pairwise nonisomorphic.

Proposition 2.4. Let $A$ be a separable unital $C^{*}$-algebra. For every $n \geqq 1$, we have $j\left(A \otimes M_{n}\right)=j(A) n$.

Proof. Let $\tau_{0}: A \rightarrow \mathscr{Q}\left(\mathscr{S}_{\mathcal{C}}\right)$ be a strongly trivial extension of $A$. Then $\tau_{0} \otimes i_{n}: A \otimes M_{n} \rightarrow \mathscr{Q}(\mathfrak{S}) \otimes M_{n}=\mathscr{Q}\left(\mathfrak{S} \otimes C^{n}\right)$ is a strongly trivial extension of $A \otimes M_{n}$, where $i_{n}: M_{n} \rightarrow M_{n}$ is the identity map. The unitaries $v$ in $\mathscr{Q}(\mathfrak{S}) \otimes M_{n}$ that commute with $\left(\tau_{0} \otimes i_{n}\right)\left(A \otimes M_{n}\right)$ are precisely those of the form $v=u \otimes I_{n}$, with $I_{n}$ the identity of $M_{n}$ and $u$ a unitary in $\mathscr{Q}(\mathfrak{S})$ commuting with $\tau_{0}(A)$. Since the index of $v$ is $n$ times the index of $u$, the proposition follows from Remark 2.1 (c).

CoROLlary 2.5. Let $G$ be the free product of two cyclic groups. Then the $C^{*}$-algebras $C_{r}^{*}(G) \otimes M_{n}(n=1,2, \cdots)$ are pairwise non- 
isomorphic.

Proof. One checks easily that $G$ can be embedded in $\boldsymbol{Z}_{p} * \boldsymbol{Z}_{p}$ for some positive integer $p$. (The case $G=\boldsymbol{Z} * \boldsymbol{Z}_{2}$ requires somewhat special treatment. Here, we may embed $G$ in $\boldsymbol{Z}_{4} * \boldsymbol{Z}_{4}$ as the subgroup generated by $b c b$ and $c^{2}$, where $b$ and $c$ are the basic generators of $\left.\boldsymbol{Z}_{4} * \boldsymbol{Z}_{4} \cdot\right)$ This means that $C_{r}^{*}(G)$ embeds in $C_{r}^{*}\left(\boldsymbol{Z}_{p} * \boldsymbol{Z}_{p}\right)$, so $j\left(C_{r}^{*}(G)\right)$ divides $p$ by Theorem 2.2. In particular, $j\left(C_{r}^{*}(G)\right) \neq 0$ and the corollary follows from Proposition 2.4.

It would be of some interest to compute $j\left(C_{r}^{*}\left(\boldsymbol{Z}_{m} * \boldsymbol{Z}_{n}\right)\right)$ for $m$, $n \geqq 2$. Since $\boldsymbol{Z}_{m} * \boldsymbol{Z}_{n}$ embeds in $\boldsymbol{Z}_{p} * \boldsymbol{Z}_{p}$, where $p$ is the least common multiple of $m$ and $n$, we know that $j\left(C_{r}^{*}\left(\boldsymbol{Z}_{m} * \boldsymbol{Z}_{n}\right)\right)$ divides $p$. Is $j\left(C_{r}^{*}\left(\boldsymbol{Z}_{m} * \boldsymbol{Z}_{n}\right)\right)$ equal to $p$ ?

\section{REFERENCES}

1. B. Blackadar, Weak expectations and nuclear $C^{*}$-algebras, preprint.

2. L. Brown, R. Douglas, and P. Fillmore, Extensions of $C^{*}$-algebras and K-homology, Ann. Math., 105 (1977), 265-324.

3. M. Choi, $A$ simple $C^{*}$-algebra generated by two finite-order unitaries, Canad. J. Math., to appear.

4. M. Choi and E. Effros, Nuclear $C^{*}$-algebras and injectivity: the general case, Indiana U. Math. J., 26 (1977), 443-446.

5. J. Cuntz, SimpleC *algebras generated by isometries, Commun. Math. Phys., 57 (1977), 173-185.

6. J. Dixmier, Les $C^{*}$-Algebres et Leurs Representations, Gauthier-Villars, Paris, 1969.

7. F. Greenleaf, Invariant Means on Topological Groups, Van Nostrand, New York, 1969.

8. M. Hall, Theory of Groups, MacMillan, New York, 1959.

9, E. C. Lance, On nuclear C*-algebras, J. Functional Analysis, 12 (1973), 157-176.

10. W. Paschke and N. Salinas, Matrix algebras over $O_{n}$, Michigan Math. J., 26 (1979), 3-12.

11. R. Powers, Simplicity of the $C^{*}$-algebra associated with the free group on two generators, Duke Math. J., 42 (1975), 151-156.

12. N. Salinas, Hypoconvexity and essentially n-normal operators, Trans. Amer. Math. Soc., to appear.

13. D. Voiculescu, A non-commutative Weyl-von Neumann theorem, Rev. Roum. Math. Pures Appl., 21 (1976), 97-118.

14. S. Wassermann, $A$ pathology in the ideal space of $L(H) \otimes L(H)$, preprint.

Received September 11, 1978. Research by both authors for this paper was partially supported by NSF Grant MCS 77-01850.

UNIVERSITY OF KANSAS

LAWRENCE, KS 66045 



\section{PACIFIC JOURNAL OF MATHEMATICS}

\section{EDITORS}

DoNALD BABBITT (Managing Editor)

University of California

Los Angeles, California 90024

HUGo Rossi

University of Utah

Salt Lake City, UT 84112

C. C. MOORE and ANDREW OGG

University of California

Berkeley, CA 94720
J. DUGUNDJI

Department of Mathematics University of Southern Californı Los Angeles, California 90007

R. Finn and J. Milgram Stanford University Stanford, California 94305

\section{ASSOCIATE EDITORS}

E. F. BECKENBACH

B. H. Neumann
F. WOLF

K. YOSHIDA

\section{SUPPORTING INSTITUTIONS}

UNIVERSITY OF BRITISH COLUMBIA CALIFORNIA INSTITUTE OF TECHNOLOGY UNIVERSITY OF CALIFORNIA MONTANA STATE UNIVERSITY UNIVERSITY OF NEVADA, RENO NEW MEXICO STATE UNIVERSITY OREGON STATE UNIVERSITY UNIVERSITY OF OREGON
UNIVERSITY OF SOUTHERN CALIFORNIA STANFORD UNIVERSITY UNIVERSITY OF HAWAII UNIVERSITY OF TOKYO UNIVERSITY OF UTAH WASHINGTON STATE UNIVERSITY UNIVERSITY OF WASHINGTON 


\section{Pacific Journal of Mathematics}

Vol. 82, No. $1 \quad$ January, 1979

Werner Bäni, Subspaces of positive definite inner product spaces of countable dimension ...................................... 1

Marilyn Breen, The dimension of the kernel of a planar set..............

Kenneth Alfred Byrd, Right self-injective rings whose essential right ideals

are two-sided

Patrick Cousot and Radhia Cousot, Constructive versions of Tarski's fixed

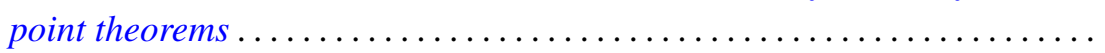

Ralph S. Freese, William A. Lampe and Walter Fuller Taylor, Congruence lattices of algebras of fixed similarity type. $I \ldots \ldots \ldots \ldots \ldots \ldots \ldots$

Cameron Gordon and Richard A. Litherland, On a theorem of Murasugi .....

Mauricio A. Gutiérrez, Concordance and homotopy. I. Fundamental

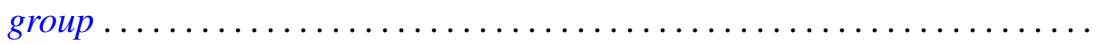

Richard I. Hartley, Metabelian representations of knot groups .............

Ted Hurley, Intersections of terms of polycentral series of free groups and free

Lie algebras ........................................

Roy Andrew Johnson, Some relationships between measures ............ 117

Oldřich Kowalski, On unitary automorphisms of solvable Lie algebras .......

Kee Yuen Lam, $K O$-equivalences and existence of nonsingular bilinear

maps...................................................

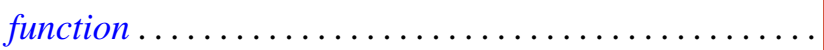

Robert A. Messer and Alden H. Wright, Embedding open 3-manifolds in compact 3-manifolds ............................

Gerald Ira Myerson, A combinatorial problem in finite fields. I . .

James Nelson, Jr. and Mohan S. Putcha, Word equations in a band of paths.

Baburao Govindrao Pachpatte and S. M. Singare, Discrete generalized Gronwall inequalities in three independent variables . .

William Lindall Paschke and Norberto Salinas, $C^{*}$-algebras associated with free products of groups ........................

Bruce Reznick, Banach spaces with polynomial norms ....

David Rusin, What is the probability that two elements of a finite group

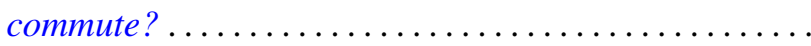

M. Shafii-Mousavi and Zbigniew Zielezny, On hypoelliptic differential operators of constant strength ...

Joseph Gail Stampfli, On selfadjoint derivation ranges .... . . .

Robert Charles Thompson, The case of equality in the matrix-valued triangle

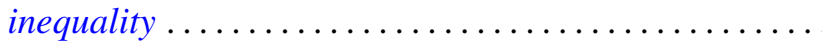

Marie Angela Vitulli, The obstruction of the formal moduli space in the negatively graded case. 